\title{
Kilogram per Cubic Meter
}

National Cancer Institute

\section{Source}

National Cancer Institute. Kilogram per Cubic Meter. NCI Thesaurus. Code C42576.

A SI derived unit of mass concentration defined as the concentration of one kilogram of a substance per unit volume of the mixture equal to one cubic meter, or the concentration of one milligram of a substance per unit volume of the mixture equal to one milliliter, or one gram of a substance per one liter of the mixture. It is also a unit of mass density (volumic mass) defined as the density of substance which mass equal to one milligram occupies the volume one milliliter. 